\title{
Technical Loss Calculation in Distribution Grids Using Equivalent Minimum Order Networks and an Iterative Power Factor Correction Procedure
}

\author{
Carlos Eduardo Moreira Rodrigues ${ }^{1, *}$, Maria Emilia de Lima Tostes ${ }^{1}$, Ubiratan Holanda Bezerra ${ }^{1}$, \\ Thiago Mota Soares ${ }^{1} \mathbb{D}$, Edson Ortiz de Matos ${ }^{1}$, Lázaro Serra Soares Filho ${ }^{2}$, Elaine Cristina dos Santos Silva ${ }^{2}$, \\ Michel Ferreira Rendeiro ${ }^{2}$ and Carlos Jeferson da Silva Moura ${ }^{2}$
}

1 Electrical Engineering Faculty, Institute of Technology, Federal University of Pará, Belém, PA 66075-110, Brazil; tostes@ufpa.br (M.E.d.L.T.); ubiratan.fapespa@gmail.com (U.H.B.); thiagosoares@ufpa.br (T.M.S.); ortiz@ufpa.br (E.O.d.M.)

2 Equatoria Energia Pará, Belém, PA 66823-010, Brazil; lazaro.filho@equatorialenergia.com.br (L.S.S.F.); elaine.santos@equatorialenergia.com.br (E.C.d.S.S.); michel.rendeiro@equatorialenergia.com.br (M.F.R.); carlos.moura@equatorialenergia.com.br (C.J.d.S.M.)

* Correspondence: carloseduardo.pa@hotmail.com; Tel.: +55-091-8268-5732

Citation: Moreira Rodrigues, C.E.; de Lima Tostes, M.E.; Holanda Bezerra, U.; Mota Soares, T.; Ortiz de Matos, E.; Serra Soares Filho, L.; dos Santos Silva, E.C.; Ferreira Rendeiro, M.;

Jeferson da Silva Moura, C. Technical Loss Calculation in Distribution Grids Using Equivalent Minimum Order Networks and an Iterative Power Factor Correction Procedure. Energies 2021, 14, 646. https://doi.org/ $10.3390 /$ en14030646

Academic Editor: Ying-Yi Hong Received: 16 December 2020

Accepted: 17 January 2021

Published: 27 January 2021

Publisher's Note: MDPI stays neutral with regard to jurisdictional claims in published maps and institutional affiliations.

Copyright: (C) 2021 by the authors. Licensee MDPI, Basel, Switzerland. This article is an open access article distributed under the terms and conditions of the Creative Commons Attribution (CC BY) license (https:/ / creativecommons.org/licenses/by/ $4.0 /)$.

\begin{abstract}
Energy losses are a fundamental issue in the electricity distribution sector, being an inevitable consequence of transporting energy from supplying sources to consumers' installations and are becoming one of the factors to be considered in planning and operation of electrical distribution networks. So, electrical distribution losses must be continuously monitored so that they are kept within acceptable levels to ensure the business profitability as well as the good power quality of supplied energy. In this context, this work introduces a modified methodology for technical losses calculation with the application of the concept of reduced equivalent networks, via definition of an Equivalent Operational Impedance, taking as a starting point the electrical network modeling in the Open Distribution System Simulator (OpenDSS). The losses calculation also considers customer's energy billing measurement data, measurements of injected energy and power factor at the feeder's coupling bus at the substation, also considering measurement campaigns to characterize the load consumption profiles for working days, Saturdays and Sundays. The proposed methodology disaggregates energy injections in billed energy, technical and non-technical losses parcels, and presents, as the results have demonstrated, a good precision in the proposed calculation procedures.
\end{abstract}

Keywords: electric distribution systems; technical losses; non-technical losses; Equivalent Operational Impedance (EOI)

\section{Introduction}

Energy losses are an inherent aspect of the electric energy distribution service, resulting from several factors, mainly due to physical phenomena that originate from the electric current flow or other factors, such as the poor management of electric utilities or unlawful actions by consumers. In this aspect, electrical losses can be classified according to their origin in technical losses and non-technical losses.

Technical losses are associated with the power dissipation in the different elements of the energy distribution system, in the processes of transformation, transport and energy measurement, representing energy losses that occur in delivering energy from the transmission system and substations to final consumers. These losses cannot be eliminated due to the associated physical phenomena, but their minimization is a constant study subject aiming at the optimization of the distribution system [1].

On the other hand, non-technical losses, determined by the difference between total losses and technical losses, correspond to the energy that is distributed and consumed, but does not return any revenue to the electrical utilities due to several factors, such as 
thefts, fraud, measurement errors, errors in the billing process and consumers without measuring equipment [2].

Technical losses are relatively known and monitored, being calculated in the operation planning studies with the objective of guiding measures to be adopted aiming at their reduction. On the other hand, non-technical losses, despite being a crucial problem for electric utilities in many countries, are not routinely addressed in power flow planning studies [3].

The overall losses in distribution systems, ideally, should vary between 3 and $6 \%$ in relation to the injected energy [4]. However, in developed countries, they are around $10 \%$, while in developing countries, the same losses can reach around $20 \%$ in the average [4]. Therefore, the reduction of these percentages through the improvement of the electrical network is fundamental for electric utilities because, the smaller the losses, the greater the profit obtained in the business, also allowing the improvement of service quality provided to consumers [5].

Given the importance of losses monitoring for technical and financial viability of the energy distribution business, it is essential to develop methodologies that return accurate results, associated with models that best represent the electric network elements for the calculation of technical and non-technical losses in order to subsidize regulatory parameters for the energy market, and for the electric utilities planning sectors.

The usual procedure for losses calculation consists in the estimation of active power losses under maximum load conditions, using computational models and load data and, subsequently, the application of a loss factor to estimate the average energy losses. Subsequently, non-technical losses are obtained by subtracting the total losses from the obtained technical losses [6,7]. Thus, the load factor is a parameter that relates the losses under system maximum loading condition with the total energy losses for a given electric network [8]. However, the adoption of this methodology inserts a certain degree of imprecision in the technical losses calculation due to two main factors: first, there is no direct relationship between maximum demand and energy losses, besides that this model must be adjusted for each electric network; second, the maximum demand for a given system varies somewhat and it is usually measured with less precision than the consumed energy in customer's facilities [8].

In order to carry out a more detailed calculation of technical losses in electrical distribution systems, a line of study was developed based on a calculation methodology by segments of the electrical distribution network, using topological data from the electric network and consumer billing, as well as measurement campaign data to obtain daily load curves [9-11]. After, the methodology was adapted to the particular case in which it considers low voltage networks when detailed data of the grid's elements are not available [12].

Currently, in addition to the methodologies already presented, the analysis of distribution systems losses tends to apply network state estimators and online power flow algorithms, with the aim of providing a more accurate calculation of feeder losses than that performed using conventional approaches [7]. Another research line proposes the calculation of technical losses through a temperature-dependent predictive model that uses data from smart meters and distribution transformers to detect energy thefts in a given area $[13,14]$.

With regard specifically to non-technical losses, for their detection and identification, most of the published work involves the development of statistical techniques and computational intelligence in order to detect irregular energy consumption patterns [15-17]. From the identification of suspected locations of non-technical losses and the estimation of their magnitudes, the methodologies already covered include them in the global balance of energy losses.

A new proposal for the calculation of technical and non-technical losses, the Equivalent Operational Impedance (EOI), was proposed in [18]. Several analyzes involving IEEE test systems and real distribution feeders, under different operating conditions, were carried out later $[19,20]$, in which it was possible to observe the good precision of the method for 
calculating losses. The same procedure was compared with the methodology for calculating regulatory losses in Brazil, established by the National Electric Energy Agency (ANEEL), through simulations in a test system and in a real distribution feeder. The comparison proved the superior accuracy of the EOI in relation to the ANEEL methodology and the low computational cost in obtaining technical and non-technical losses [21].

Based on the Equivalent Operational Impedance proposal for the calculation of technical and non-technical losses in distribution systems, this work presents a methodology for applying it to a real distribution feeder, taking as a starting point the network modeling in the Open Distribution System Simulator (OpenDSS), considering the customer's billing measurement data, data of feeder's injected energy and power factor measured at the coupling bus with the substation and data of measurement campaigns to characterize the load consumption for working days, Saturdays and Sundays. With all these data, it is possible to calculate technical losses due to supplying regular loads, non-technical losses and also technical losses due to supplying non-technical losses.

Associated with the EOI methodology application, this paper also presents as a contribution an algorithm that aims to adjust the loads power factors in such a way that the calculated power factor at the feeder coupling bus with the substation through the load flow algorithm, is consistent with the measured power factor value at the same bus. In summary, the proposal presented is an iterative process with the purpose of matching the calculated power factor to the measured one, at the feeder coupling bus with the substation, by adjusting the power factor of all loads, with the purpose of gaining precision in technical loss calculation in the electrical network.

\section{Methodology for Calculation of Technical Losses}

The calculation methodology proposed in this work is based on three main points: the electric network modeling, with all its constituent elements, in the OpenDSS software and the use of its load flow calculation methodology; the use of Operational Equivalent Impedance for the calculation of total technical losses; and the implementation of a power factor correction algorithm for low voltage loads, a factor that significantly influences the calculation of technical losses.

\subsection{Network Modeling}

The proposed loss calculation methodology is based on a detailed network modeling, using software OpenDSS, covering all distribution network elements, from the substation power transformers to the terminal branches, in which low voltage consumers are connected. In this way, all the necessary parameters for the network's elements modeling were taken from two main sources: the first concerns the BDGD (The Utility Georeferenced Database), which contains geographical, electrical and commercial parameters regarding the network elements; and the second data source comes from measurement campaign data to characterize loads within the scope of the periodic tariff review process.

\subsubsection{Utility Georeferenced Database}

In the Brazilian electric system, ANEEL, through Module 10 of PRODIST (Electricity Distribution Procedures in the National Electric System), determined the distribution electric utilities to create and maintain a database to standardize the information availability on network elements by type, technical data and locations, to increase reliability and traceability of this information in the periodic tariff review process [22].

Table 1 presents the information taken from the utility's database for the electric network modeling, according to all its constituent elements, according to the segment to which they belong: substation transformers; medium voltage network with capacitor banks and associated medium voltage loads; distribution transformers; low voltage network; low voltage branch circuits; and low voltage loads. 
Table 1. Electric network modeling using BDGD data.

\begin{tabular}{|c|c|}
\hline Element & Data \\
\hline Substation transformer & $\begin{array}{c}\text { Electrical connection point, number of windings, rated power, } \\
\text { tap, resistance and reactance }\end{array}$ \\
\hline Medium voltage line & $\begin{array}{c}\text { Electrical connection point, number of phases, length, } \\
\text { electrical parameters of cable }\end{array}$ \\
\hline Medium voltage load & $\begin{array}{c}\text { Electrical connection point, number and sequence of phases, } \\
\text { billed energy, load curve typology }\end{array}$ \\
\hline Medium voltage capacitor bank & Electrical connection point, rated power \\
\hline Distribution transformer & $\begin{array}{c}\text { Electrical connection point, number of windings, rated power, } \\
\text { tap, resistance and reactance }\end{array}$ \\
\hline Low voltage line & $\begin{array}{l}\text { Electrical connection point, number of phases, length, } \\
\text { electrical parameters of cable }\end{array}$ \\
\hline Low voltage branch circuit & $\begin{array}{l}\text { Electrical connection point, number of phases, length, } \\
\text { electrical parameters of cable }\end{array}$ \\
\hline Low voltage load & Electrical connection point \\
\hline
\end{tabular}

For accessing BDGD, a computational tool was developed in order to read the relevant information from network elements to describe them in the OpenDSS programming language and perform the electrical connection, thus creating a concise circuit compatible with the simulation software.

\subsubsection{Measurement Campaigns}

The second main data source for the electric network modeling are measurement campaigns to obtain load curves to better reproduce daily consumption of distribution customers, according to their usage electricity habits. In Brazil, it is a common practice of electric energy utilities to carry out extensive measurement campaigns to characterize the loads in their area within the scope of the tariff review process, defined by the national regulator. Such measurement campaigns cover all consumers classes according to their tariff group and voltage level and constitute demand data obtained at 15-min intervals (96 points over a measurement day), comprising characteristic consumption curves for a working day, a Saturday and a Sunday for the same consumer type. The load curves obtained in this process were then also used in methodologies for calculating technical losses.

Using the data contained in the measurement campaigns, load curves were obtained, which characterize the energy consumption of a consumer's facility throughout the day, to be used in the simulations. The load curves are contained in the measurement campaign according to the voltage level and the tariff group to which the loads belong. Table 2 shows the number of types of load curves in the measurement campaign, covering medium and low voltage consumers and residential, commercial, industrial, and rural consumers.

Table 2. Load curves typologies for load modelling.

\begin{tabular}{cc}
\hline Tariff Subgroup & Typologies of Load Curve \\
\hline Residential low voltage & 10 \\
Commercial low voltage & 10 \\
Industrial low voltage & 10 \\
Rural low voltage & 10 \\
Street lighting & 1 \\
Medium Voltage & 10 \\
\hline
\end{tabular}

Still using the measurement campaigns, multiplicative correction factors were implemented for the average load demand, obtained from energy bills, applied to each load, according to its tariff subgroup and load curve typology. For this, the same data obtained from the measurement campaigns were used to characterize the loads. As a first step, billed energy data were obtained for all loads for which load curves were also specified. 
The energy data comprise the energy consumed by the load for a working day, a Saturday, and a Sunday:

$$
e_{\text {load }} u, s, d=\sum D_{i} u, s, d \cdot \Delta t
$$

where:

$e_{\text {load }} u, s, d$-Energy consumed for one working day, Saturday and Sunday for the same load [kWh];

$D_{i}$ - Instant load curve demand [kW];

$\Delta t$-Daily load curve interval duration [h].

Subsequently, in order to calculate the average monthly energy for loads, the energies obtained in (1) were used, relating them to the number of working days, Saturdays, Sundays and the total days of the respective month for which the calculation of energy loss is performed.

$$
e_{\text {mean }}=\frac{\left(e_{\text {load }} u \cdot n_{u}\right)+\left(e_{\text {load }} s \cdot n_{s}\right)+\left(e_{\text {load }} d \cdot n_{d}\right)}{n_{\text {days }}}
$$

where:

$e_{\text {mean }}$-Average monthly load energy [kWh];

$e_{\text {load }} u$-Energy consumed for one business day [kWh];

$e_{\text {load }} s$-Energy consumed for a Saturday [kWh];

$e_{\text {load }} d$-Energy consumed for a Sunday [kWh];

$n_{u}$-Number of working days of the month;

$n_{s}$-Number of Saturdays of the month;

$n_{d}$-Number of Sundays of the month;

$n_{\text {days }}-$ Number of days of the month;

Finally, the average load demand multiplicative factors are obtained by means of the ratio between the daily energies calculated for each specified load and their respective average monthly energy.

$$
f_{\text {load }} u, s, d=\frac{e_{\text {load }} u, s, d}{e_{\text {mean }}}
$$

where:

$f a c_{\text {load }} u, s, d$-Correction factors for load consumption for one business day, Saturday and Sunday;

$e_{\text {load }} u, s, d$-Energy consumed for one working day, Saturday and Sunday for the same load [kWh];

$e_{\text {mean }}$-Average monthly load energy [kWh];

As a result of the used methodology, Figure 1 presents three load curves that describe consumption characteristic of a residential (Figure 1a), commercial (Figure 1b) and industrial (Figure 1c) consumer. In the figure, the vertical axis variable is the multiplier factor of the average load demand for each instant of the load curve. It can be seen that the curves applied to each low or medium voltage load, depending on the tariff group and voltage level, make the distinction, both in form and in billed energy, for the same load on different days of the week. 


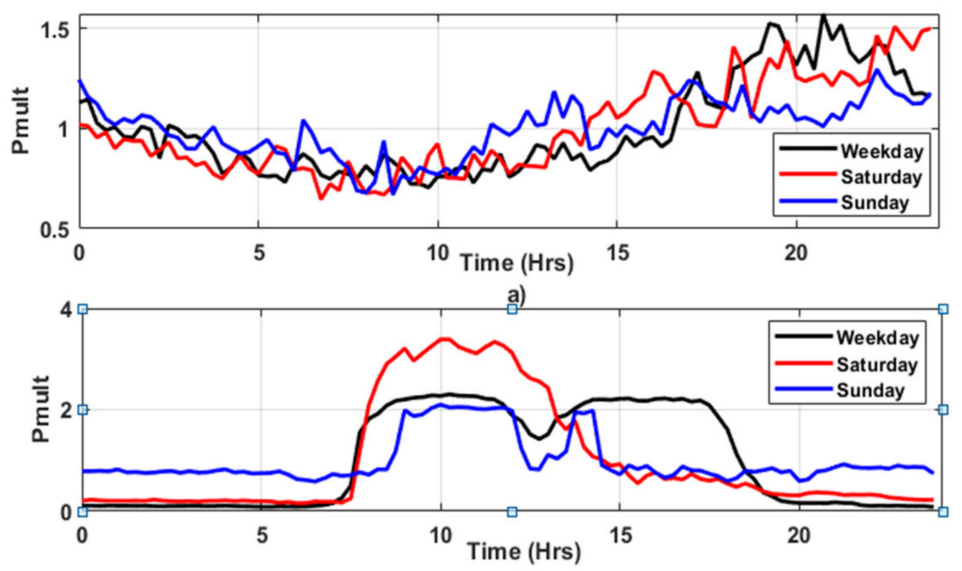

b)

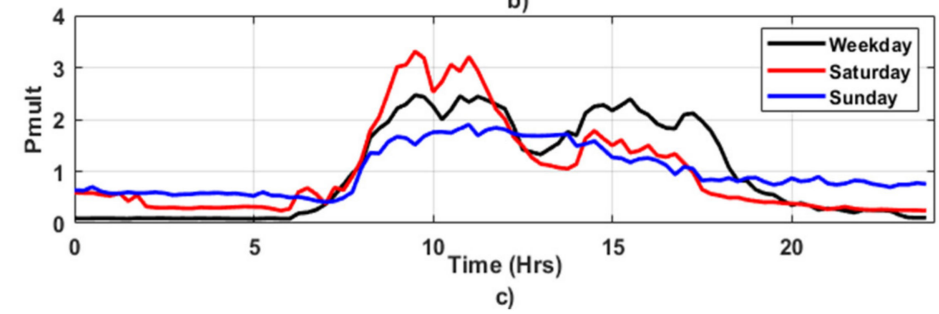

Figure 1. Typical load curves for; (a) residential consumer; (b) commercial consumer; (c) industrial consumer for one business day (black), one Saturday (red) and one Sunday (blue).

\subsection{Equivalent Operational Impedance}

Equivalent Operational Impedance (EOI) is defined as the complex functional relationship between the total loss of an electrical network divided by the square rms value of the injected electric current at the point of interest for establishing the network equivalent. In radial distribution grids for example, the point of interest can be the feeder's coupling bus at the substation, and in this case, the EOI represents the whole feeder, including the medium and low voltages grids. If the point of interest is a low voltage distribution transformer, then the EOI represents the corresponding low voltage grid. Therefore, Equivalent Operational Impedance is defined as [19,20]:

$$
Z_{E O I}=R_{E O I}+j X_{E O I}=\frac{P_{T}}{I_{i n j}^{2}}+j \frac{Q_{T}}{I_{i n j}^{2}}
$$

where:

$Z_{E O I}$-Equivalent Operational Impedance (EOI) [Omhs];

$R_{E O I}$-Equivalent Operational Resistance [Omhs];

$X_{E O I}$-Equivalent Operational Reactance [Ohms];

$P_{T}$-Total active technical loss calculated to the equivalent network [kW];

$Q_{T}$-Total reactive technical loss calculated to the equivalent network [kVar];

$I_{i n j}$-RMS injected current in the point of interest [A];

In this way, the defined EOI represents a minimal order operational equivalent with the specific purpose of calculating technical losses, the value of which depends on the total calculated losses and the square of the injected rms current. Total losses, in turn, are related to the electrical network topology, the supplied electrical load and the voltage magnitude for an operating point under analysis [18]. Therefore, for each network operational condition, an EOI can be defined, which reflects its operation.

However, taking the definition of the EOI resistive $\left(R_{E O I}\right)$ and reactive $\left(X_{E O I}\right)$ components $\left(R_{E O I}=\frac{P_{T}}{I_{i n j}^{2}}\right)$ and $\left(X_{E O I}=\frac{Q_{T}}{I_{i n j}^{2}}\right)$, it is concluded that the load current influences both the numerator, and the denominator, once $P_{T}$ and $Q_{T}$ are given as: $\left(P_{T}=R \cdot I_{i n j}^{2}\right)$ 
and $\left(Q_{T}=X \cdot I_{i n j}^{2}\right)$,. Thus, it is reasonable to expect a cancellation effect due to the load current and, consequently, that $R_{E O I}$ and $X_{E O I}$ are not very sensitive to loading variations. Therefore, for variable network loading conditions, but maintaining the same network topology and adequate voltage profile for all loading conditions, for example voltage magnitude variation between $\pm 5 \%$, the EOI is expected to have an approximately constant value for all loading conditions [18-21].

Also, due to the natural imbalance in distribution networks, in which the loading among phases can vary substantially, a three-phase Equivalent Operational Impedance can also be defined, which is calculated from the relation between the network total loss and sum of the squares of injected currents in each phase [19], according to (5)-(7):

$$
Z_{E O I \_3}=R_{E O I \_3}+j X_{E O I \_3}
$$

where:

$\mathrm{Z}_{E O I \_3}$-Equivalent Operational Three-Phase Impedance (EOI) [Omhs];

$R_{E O I_{3} 3}-$ Equivalent Operational Three-Phase Resistance [Omhs];

$\mathrm{X}_{\mathrm{EOI} \_3}$-Equivalent Operational Three-Phase Reactance [Ohms];

$$
\begin{aligned}
& R_{E O I_{-} 3}=\frac{P_{T_{-} A+} P_{T_{-} B}+P_{T_{-} C}}{I_{\text {inj_A }}^{2}+I_{\text {inj_B }}^{2}+I_{\text {inj_C }}^{2}}=\frac{P_{T}}{I_{\text {inj_A } A}^{2}+I_{\text {inj_B }}^{2}+I_{\text {inj_C }}^{2}} \\
& X_{E O I_{-} 3}=\frac{Q_{T_{-} A+} Q_{T_{\_} B}+Q_{T_{-} C}}{I_{\text {inj_A }}^{2}+I_{\text {inj_B }}^{2}+I_{\text {inj_C }}^{2}}=\frac{Q_{T}}{I_{\text {inj_A } A}^{2}+I_{\text {inj_B }}^{2}+I_{\text {inj_C } C}^{2}}
\end{aligned}
$$

where:

$P_{T_{-} A, B, C}$-Active technical loss in phases $\mathrm{A}, \mathrm{B}$ and $\mathrm{C}$, respectively $[\mathrm{kW}]$;

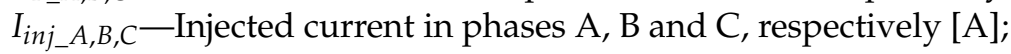

$Q_{T_{-} A, B, C}$ - Reactive technical loss in phases $\mathrm{A}, \mathrm{B}$ and $\mathrm{C}$, respectively [kVar];

The loss calculation in electric distribution networks has an additional difficulty due to the unbilled energy consumption, which is responsible for non-technical losses. However, non-technical losses generate additional technical losses, and these are difficult to calculate because unbilled energy consumptions are not known with good accuracy. In general, one can write the total active and reactive technical losses of electrical systems as being,

$$
\begin{gathered}
P_{T}=P_{T_{-} U C}+P_{T_{-} N T} \\
Q_{T}=Q_{T_{-} U C}+Q_{T_{-} N T}
\end{gathered}
$$

where:

$P_{T}$ - Total active technical loss [kW];

$P_{T_{-} U C}$-Active technical loss due to billed energy consumption [kW];

$P_{T_{-} N T}-$ Active technical loss due to unbilled energy consumption $[\mathrm{kW}]$;

$Q_{T}$-Total reactive technical loss [kVAr];

$Q_{T_{-} U C}$-Reactive technical loss due to billed energy consumption [kVar];

$Q_{T_{-} N T}$ - Reactive technical loss due to unbilled energy consumption [kVar];

Losses $P_{T_{-} U C}$ and $Q_{T_{-} U C}$ can be obtained by calculating a load flow to the electric grid considering typical loading curves for costumers' installations as obtained from the customers' energy bills, adjusting the respective power factors, according to the procedure presented in Section 2.3.

Losses $P_{T}$ and $Q_{T}$, corresponding to total losses, can be obtained for each point of the feeder loading curve, by the corresponding injected current measurement in the substation-feeder coupling bus, using the operational equivalent impedance of the respective electric grid under analysis, that is, $P_{T}=R_{E O I} \cdot I_{i n j}^{2}$ and $Q_{T}=X_{E O I} \cdot I_{i n j}^{2}$. Thus, using Equations (8) and (9), non-technical losses are calculated for each point of the feeder loading curve. 
Therefore, considering the problem presented, of determining the total technical losses, EOI can be used according to the flowchart presented in Figure 2, considering two applications:

- $\quad$ First one (Figure 2a): refers to calculation of only one EOL value which is used for all load curve points. This is justified, due to the fact the EOI behavior is little dependent on the load variation along the system load curve, provided that for all operation points voltages are kept adequate, for example, with maximum variations of up to $\pm 5 \%$ In these cases, the EOI calculated for a load curve operation point can be used for all other points with good accuracy. This strategy is adopted in references [18-21].

- Second one (Figure 2b): refers to the new approach of this paper, in which an EOI value is calculated for each operation point following the network loading curve. In this case, the EOI values $\left(Z_{E O I_{1}}, Z_{E O I_{2}}, Z_{E O I_{3}}, \ldots, Z_{E O I_{n}}\right)$ are calculated for each simulation point in OpenDSS daily mode. Therefore, considering each simulation point every $15 \mathrm{~min}, 288$ different values of EOI will be used: 96 values for a weekday, 96 for a Saturday and 96 for a Sunday.

START

(1)

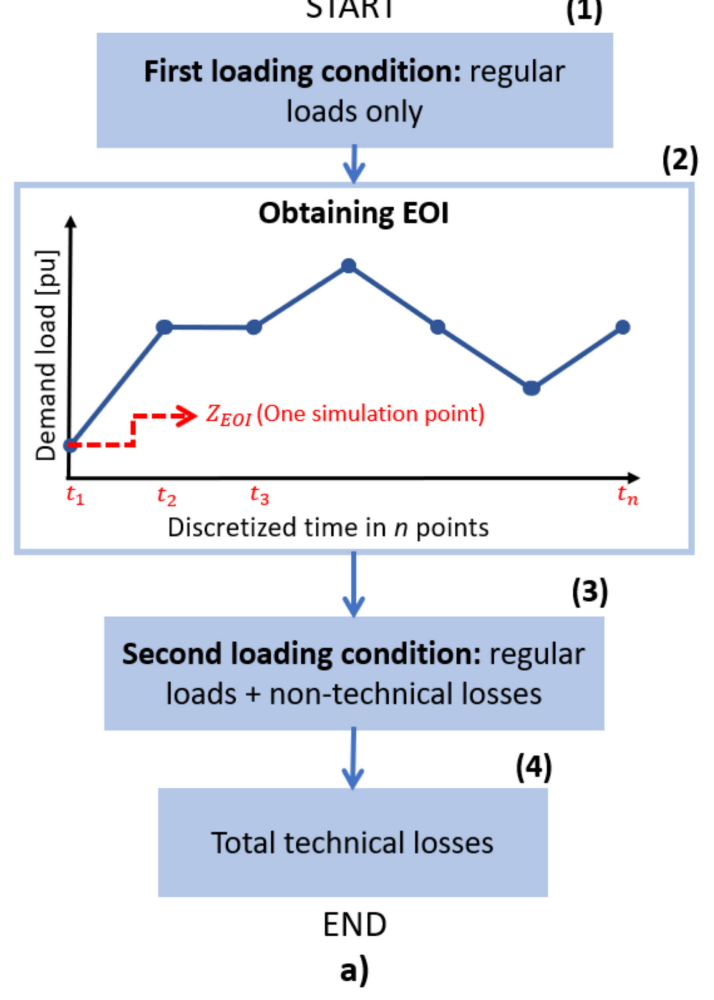

START

(1)

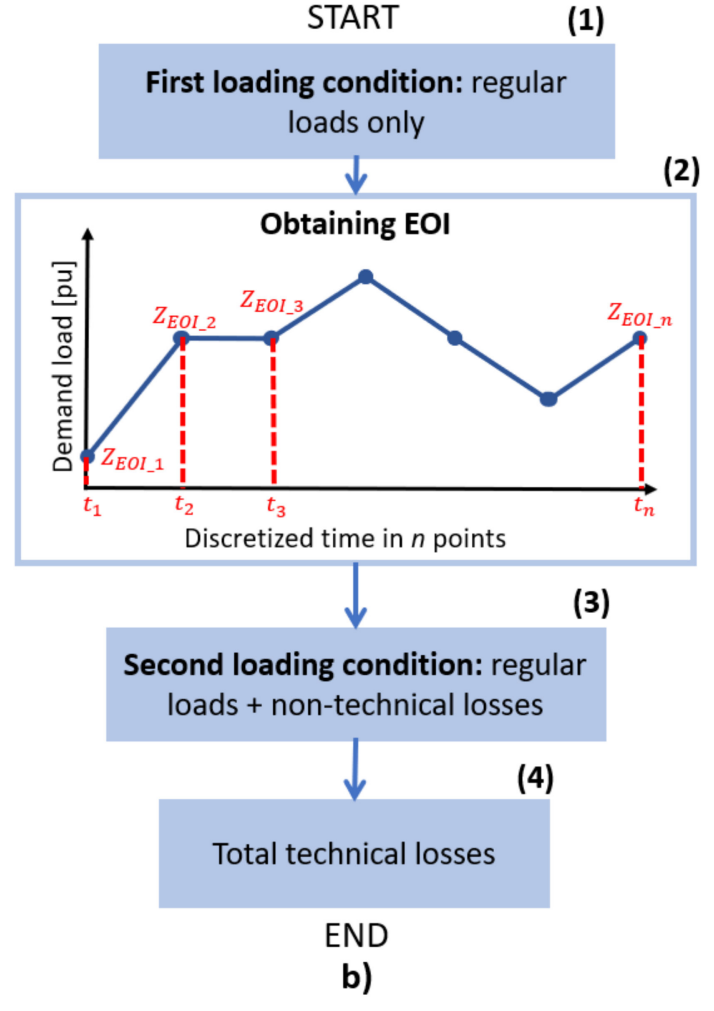

Figure 2. Methodology for applying the EOI to calculate losses. (a) one value of EOI and (b) $n$ values of EOI, according to number of loadshape points.

So, considering both applications and the flowcharts of Figure 2a,b:

(1) Initially, for a given loading condition considering only billed energy consumptions a load flow algorithm is run for the electric grid, to calculate $P_{T_{-} U C}$ and $Q_{T_{-} U C}$;

(2) For the load flow solution in (1) the EOI is calculated;

(3) Measured injected current in second loading condition, with non-technical losses, is obtained.

(4) Using the measured injected current for the loading condition under analysis, and the calculated EOI in (2), total active and reactive technical losses are obtained; 


\subsection{Algorithm for Low Voltage Load Power Factor Adjustment}

When calculating technical losses through load flow studies in the low voltage grid, an adequate load modeling procedure is essential for obtaining precise results. Thus, among the necessary parameters for load characterization is power factor that has different ranges of values for different types of consumers in the energy distribution network.

The load power factor determination directly implies the reactive power demand to be supplied by the source or by the reactive compensation elements distributed along the electric grid which impact on the electric current flowing in the distribution lines and, consequently, on the magnitude of the observed technical losses. Thus, there is a sensitive relationship between power factor and technical losses occurrence [23,24].

However, for low voltage consumers the electric utilities commercial databases usually store only information regarding the consumption of active energy for billing purposes, as these customers, in general, still do not follow stricter legislation regarding reactive energy consumption. Therefore, the power factor consideration in calculating losses in the low voltage grid implies a certain level of approximation, since it would be necessary to measure power factor at all low voltage load points, considering the different times of the day.

To solve this problem, several studies have already been carried out in order to arrive at a power factor to be used in calculating load flow that best represents the electric network reality, with an approximation level that returns accurate results in the process of determining losses. The most traditional approach consists on considering an approximate power factor that reflects the characteristics of consumers' equipment in operation for the electric grid under analysis [25] or using power factors considered typical for low voltage consumers [26-29].

Based on the literature, an algorithm was developed for adjusting power factor of low voltage distribution loads in such a way that the load flow calculated power factor at the substation-feeder coupling bus, be consistent with the power factor measured value at the same point. In summary, the proposal presented deals with an iterative process with the purpose of matching the calculated power factor to the measured one, by adjusting the low voltage loads power factors, with the purpose of gaining precision in technical losses calculation.

In this sense, an algorithm was implemented to estimate the power factor of low voltage consumers, based on the power factor value measured at the feeder-substation coupling point that is usually measured by the electric utility. Figure 3 presents a flowchart to explain the algorithm:

\section{Power factor adjustment algorithm:}

Step 1-The electric network model is loaded in OpenDSS;

Step 2-A load flow study is executed in OpenDSS in normal mode, that is, the load flow is run for the system average load condition. Initially, it was considered that all low voltage loads have a power factor equal to 0.92 , configured as an initial value. For other loads, consisting of medium voltage loads, the measured value is maintained;

Step 3-From the load flow results in step 2, the power factor at the feeder-substation coupling bus is calculated, according to Equations (10) and (11):

$$
\begin{gathered}
S_{i n j}=\sqrt{P_{i n j}^{2}+Q_{i n j}^{2}} \\
P F_{\text {calc }}=\frac{P_{i n j}}{S_{i n j}}
\end{gathered}
$$

where:

$S_{i n j}$-Total calculated apparent power injected into the feeder [kVA];

$P_{i n j}$-Total calculated active power injected into the feeder [kW];

$Q_{i n j}$-Total calculated reactive power injected into the feeder [kVAr];

$P F_{\text {calc }}$-Calculated power factor at the beginning of the feeder. 
Step 4-Having the calculated power factor in step 3, it is compared with the measured power factor provided by the substation's measurement system, thus generating a difference as in (12):

$$
\text { dif }=P F_{\text {calc }}-P F_{\text {mea }}
$$

where:

dif-Difference between calculated and measured power factors;

$F P_{\text {calc }}$-Calculated power factor;

$F P_{\text {mea }}$-Measured power;

Step 5-The calculated power factor difference is checked with the solution tolerance, chosen as 0.000001;

Step 6-If the calculated difference is less than the stipulated tolerance, the new power factors calculated by the algorithm will be assigned to the low voltage loads for loss calculation.

Step 7-However, if the difference is greater than the specified tolerance, a new power factor is assigned to the loads, given by subtracting the old power factor by the difference calculated in step 4 . The algorithm returns to step 2 again, to run the load flow algorithm with the new power factor for all low voltage loads, repeating the process until the difference between the calculated and measured power factors at the feeder-substation coupling bus is less than the stipulated tolerance.

This proposed algorithm seeks to add higher precision to technical losses calculation in electric distribution network, given the sensitive relationship between loss calculation and the choice of the load power factor.

The methodology for defining power factors for low voltage loads for loss calculation can also be implemented considering a substation composed of several connected feeders, provided that each feeder has an active and reactive energy measurement system. For this purpose, a computational routine was implemented in the OpenDSS COM interface that allows tracking all low voltage loads connected to each feeder. Based on this information, the algorithm in Figure 3 was applied to each feeder to define the power factor to be used in the loss calculation procedure.

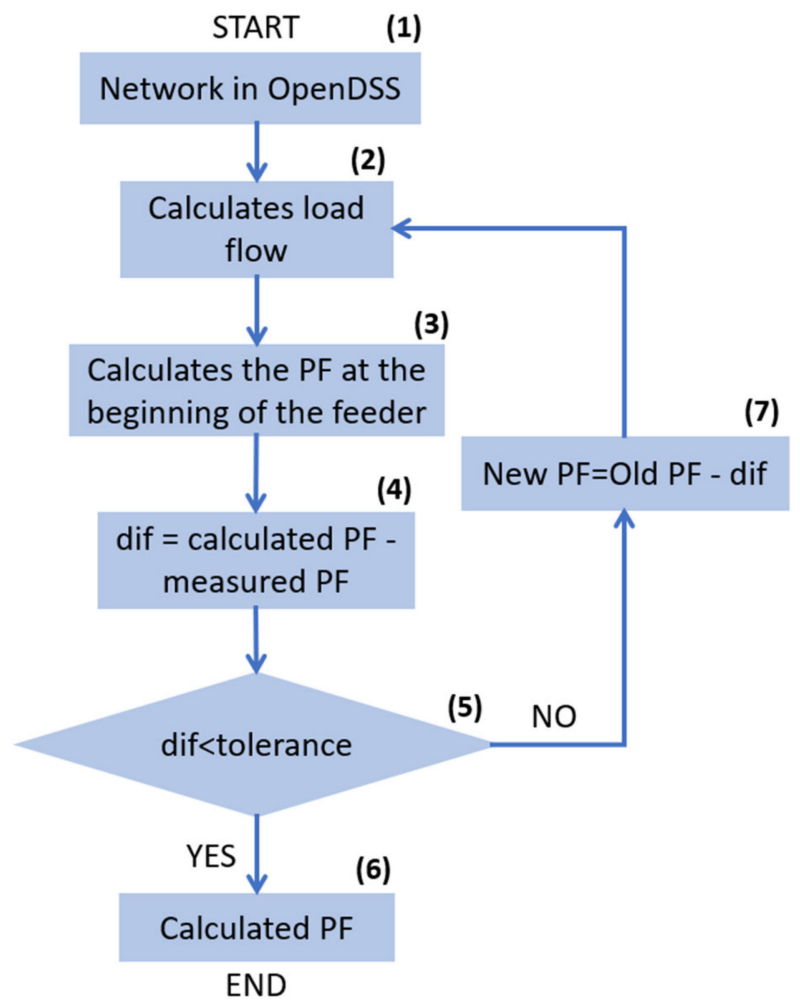

Figure 3. Flowchart of the load power factor adjustment algorithm. 
To illustrate this aspect, Figure 4 presents a generic substation with $\mathrm{n}$ feeders, for which power factors measurements (PF n) at the coupling points with the substation are available. Each feeder supplies a set of low voltage loads (LV Load $n$ ) for which new power factor values (New PF LV Load n) will be adjusted, according to the algorithm proposal presented in the flowchart in Figure 3.

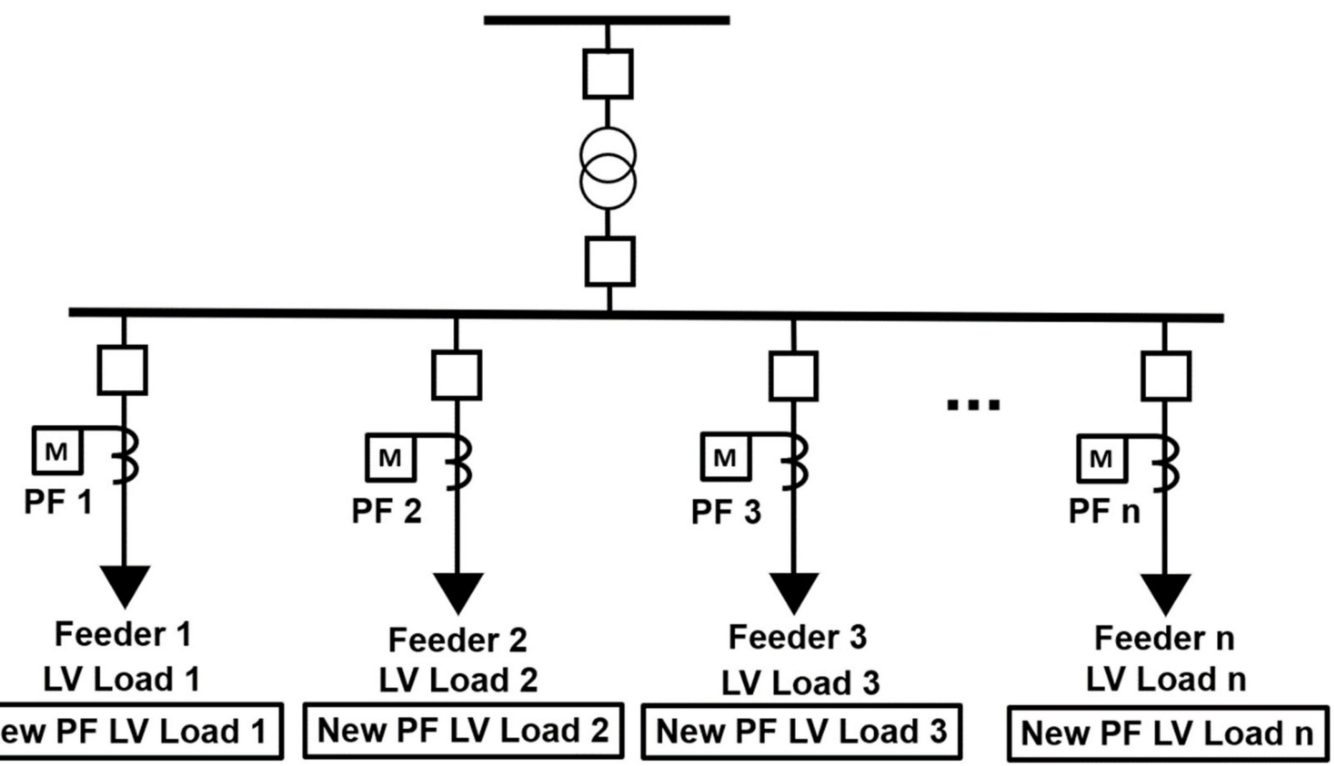

Figure 4. Illustration of applying methodology for power factor adjustment to all substation's feeders.

\subsection{Integrated Loss Calculation Methodology}

Using the network modeled in OpenDSS, the defined Equivalent Operational Impedance and the power factor adjustment algorithm for low voltage loads, the integrated methodology for calculating technical and non-technical losses was implemented, as shown in Figure 5.

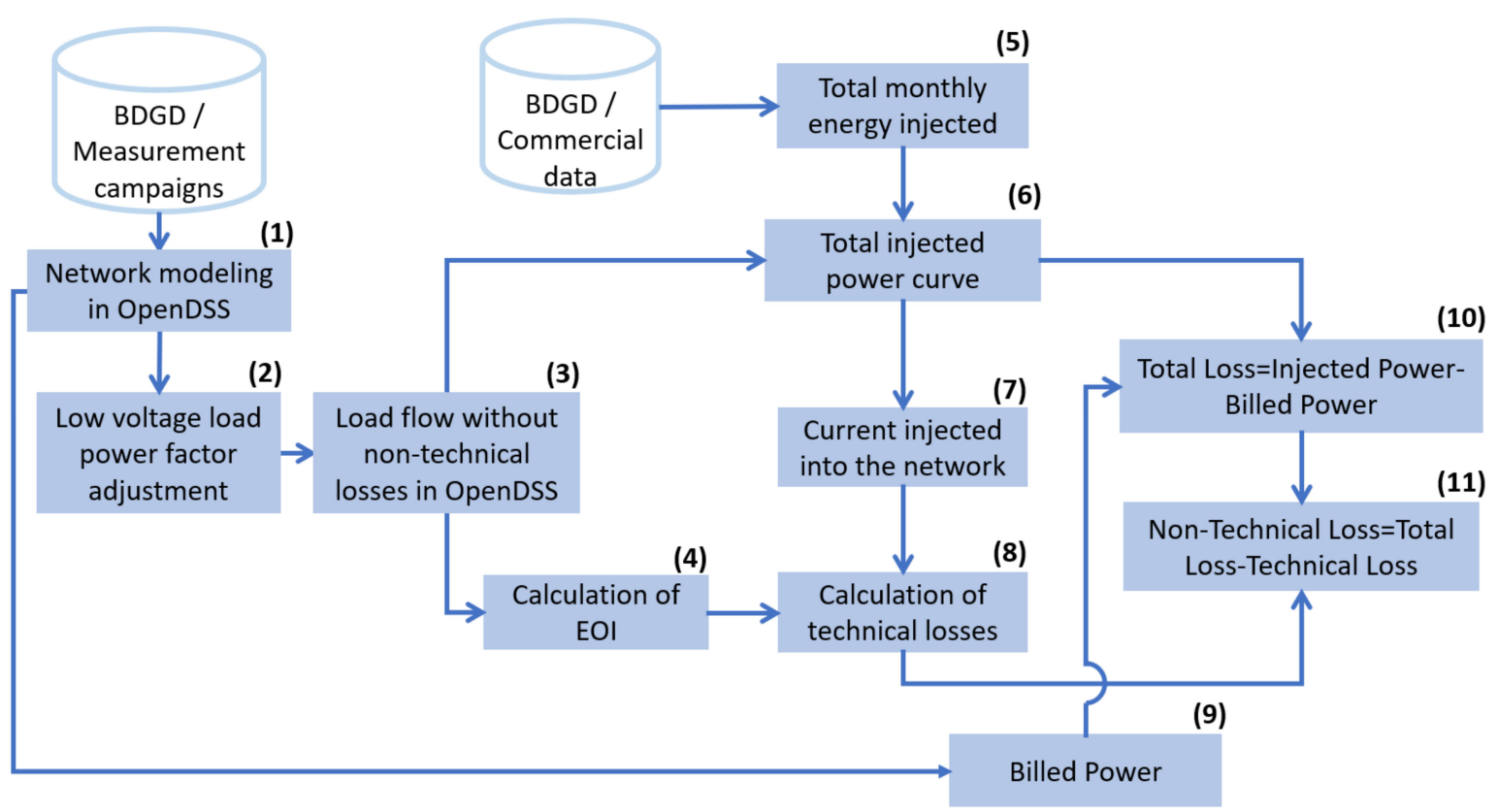

Figure 5. Flowchart of the load power factor adjustment algorithm. 


\section{Flowchart description:}

(1) Network modeling in OpenDSS is performed using the BDGD database and load curves of measurement campaigns;

(2) The algorithm for low voltage loads power factor adjustment is executed;

(3) Using the modeled network, a load flow study is carried out following typical daily load curves for a business day, a Saturday and a Sunday, specified for each individual load, with a simulation step of 15 min between load flow case studies;

(4) The Equivalent Operational Impedance (EOI) is calculated using (4);

(5) Accessing the BDGD, monthly injected energy is calculated for the feeder or substation;

(6) The total inject power curve is calculated using the value of monthly energy, obtained in (5), and the system's load curve, obtained in (3). At this point, the injected power curve considers regular and irregular loads;

(7) Total injected currents are obtained at every $15 \mathrm{~min}$ intervals from data available in (9) and voltages and power factors obtained in (10), according to relationship (13):

$$
I=\frac{P}{\sqrt{3} \cdot V \cdot F P}
$$

where:

I-Total injected current [A];

$P$-Total three-phase injected active power $[\mathrm{kW}]$;

$V$-Line voltage measurement at the feeder-substation coupling point in [kV];

$F P$-Power factor measurement at the feeder-substation coupling point.

(1) Total three-phase technical losses calculation is then carried out, which at this point includes also technical losses resulting from the supplied billed and non-billed energy.

$$
P_{T}=R_{I E O \_3} \cdot I^{2}
$$

where:

$P_{T}$-Total three-phase active technical losses in [kW];

$R_{E O I \_3}$ - Resistive portion of the Three-Phase Equivalent Operational Impedance [Ohms]; I-Total injected current [A];

(2) The total billed power of connected loads is read, at 15 min intervals, which corresponds to the respective consumed energy in this time interval. The active power of each load is given by Equation (15).

$$
D_{i}=D_{m} \cdot L S_{i} \cdot f a c_{\text {load }} u, s, d
$$

where:

$D_{i}$-Active power demand for load at time $i[\mathrm{~kW}]$;

$D_{m}$-Average load demand in [kW];

$L S_{i}$ - Multiplying factor of the load curve at time $i$;

$f a c_{\text {carga }} u, s, d$-Load consumption correction factor for one business day, Saturday and Sunday;

(3) Total system losses are calculated, from the total injected active power curve, obtained in (6), and from the billed power, obtained in (9), according to Equation (16):

$$
\text { Total Loss }=\text { Injected Power }- \text { Billed Power }
$$

(4) Finally, non-technical losses are calculated from the difference between total losses, obtained in (10), and technical losses, obtained in (8). 


\section{Results and Discussion}

In order to demonstrate the proposed methodology accuracy in calculating losses in electrical network, two electrical systems will be used, being the IEEE 123 bus test system and a real urban distribution substation in Belém City, state of Pará, Brazil, belonging to Equatorial Pará, the local electric utility.

\subsection{IEEE- 123 Test Feeder}

The IEEE- 123 system shown in Figure 6 is a distribution system with nominal voltage of $4.16 \mathrm{kV}$ and being complex enough for the evaluation of different loss scenarios. It has typical elements of real systems, such as overhead and underground lines, voltage regulators, capacitor banks and switching devices.

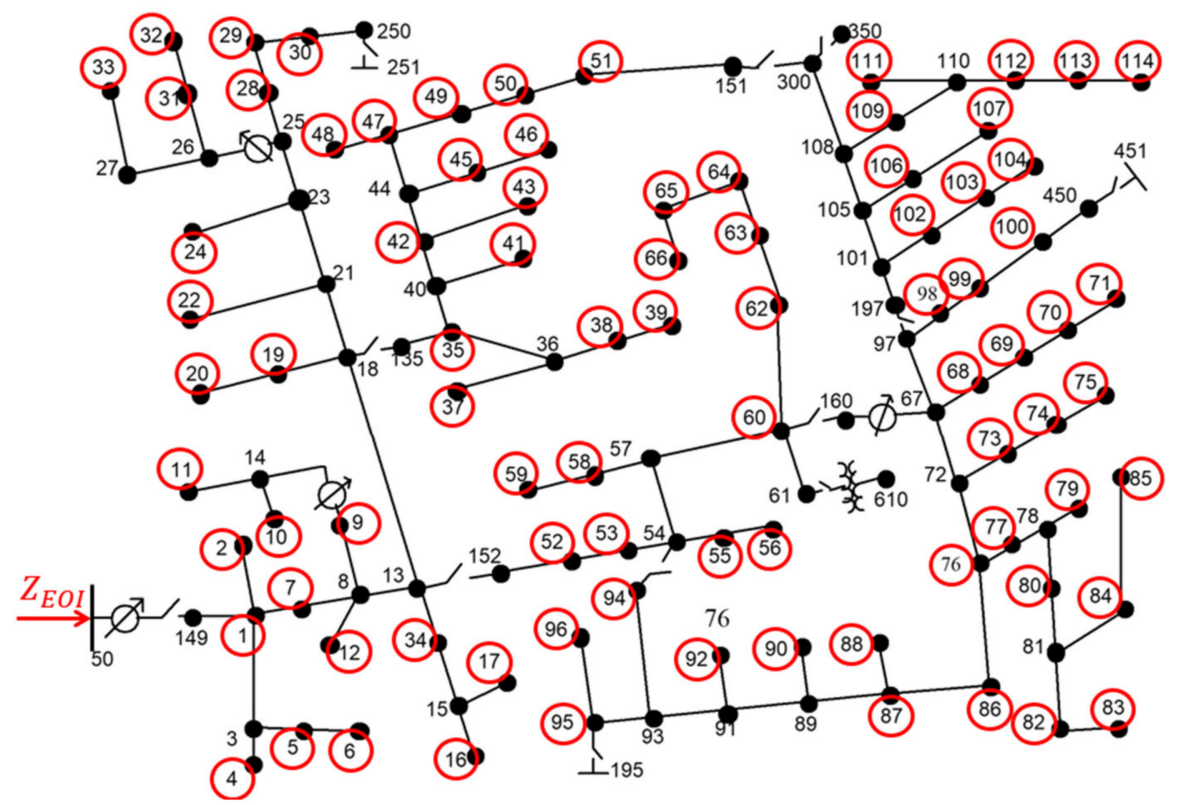

Figure 6. Allocation of non-technical losses in IEEE- 123 system [30].

Considering the original system, non-technical losses were added randomly to the load buses represented in red circles in Figure 6, in a proportion varying between 0 and $40 \%$ with respect to the given base loads. Following this procedure, total injected active power curves for a time interval of $24 \mathrm{~h}$, were obtained as shown in Figure 7, for the cases of considering only regular (billed) energy consumption, and irregular consumption, that is, with non-technical losses included.

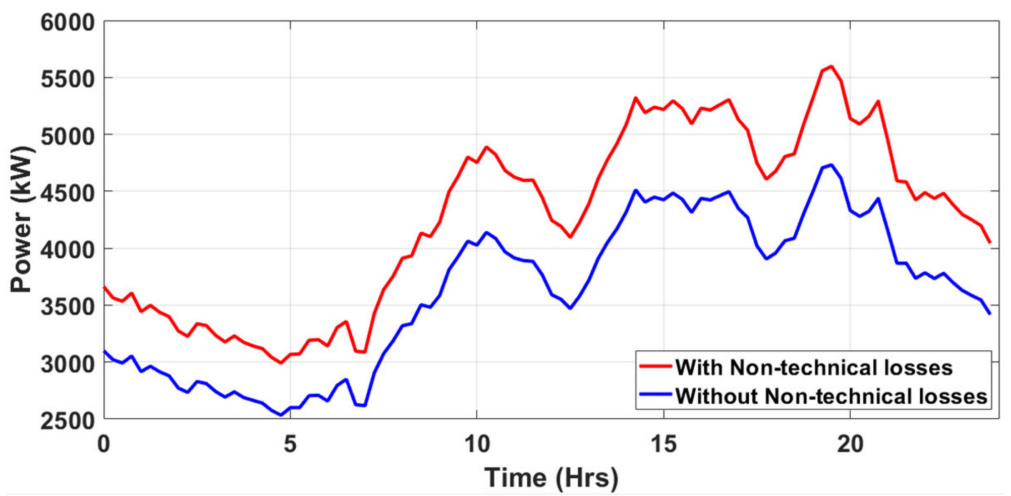

Figure 7. Injected active power in the IEEE- 123 bus test system under both loading conditions: without irregular loads (blue) and with irregular loads (red). 
For the simulation scenario presented in Figure 6, the voltage regulator transformers' taps in the IEEE 123 bus test system were maintained fixed, for the two loading conditions presented in Figure 7. This way, due to the load increase when non-technical losses are include, it implies in higher voltage drops along the system loading curve, as can be seen in Figure 8, which shows the voltage profiles for the most loaded condition at 7:45 pm, as seen in Figure 7. Considering that OpenDSS methodology considers each bus phase as a node, Figure 8 shows all IEEE system nodes.

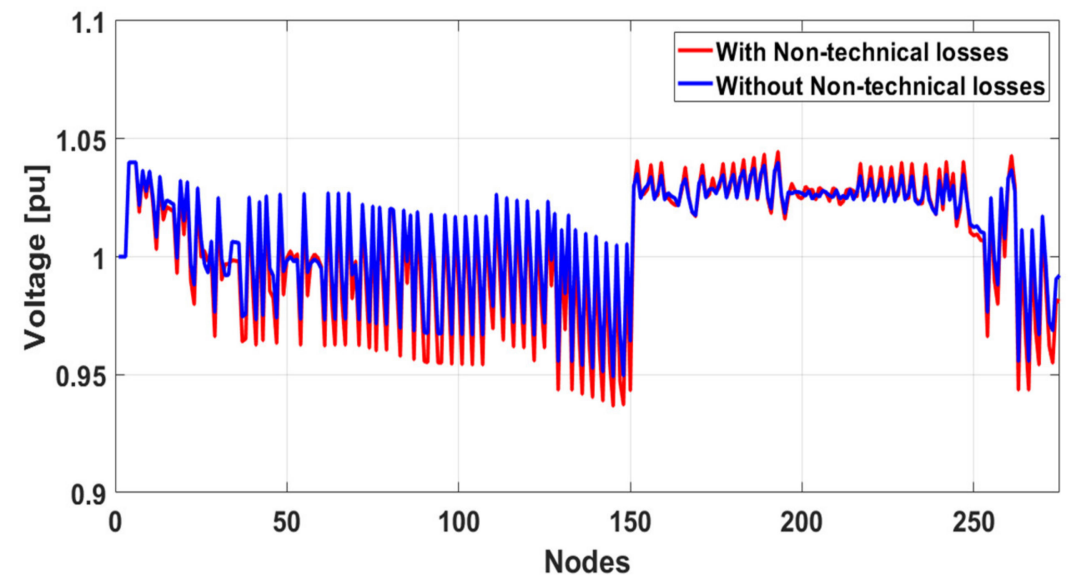

Figure 8. IEEE 123-bus test system voltage profiles at all nodes for the most loaded condition at 7:45 pm, considering non-technical losses (red) and without non-technical losses (blue).

Considering the daily mode simulation in the OpenDSS, an EOI curve was calculated representing each loading condition, discretized in 15-min intervals, totaling 96 intervals, as shown in Figure 9.

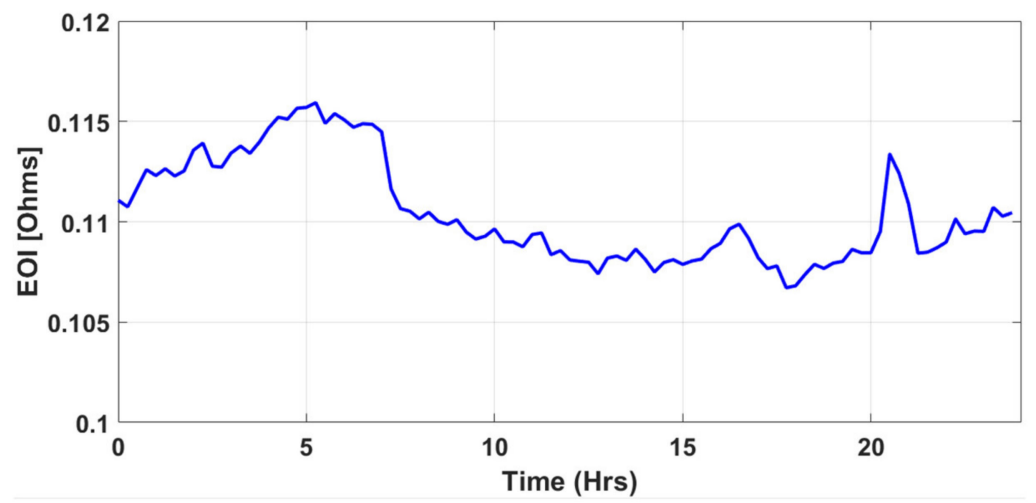

Figure 9. EOI curve over a day to calculate the total technical losses for the loading condition having irregular loads.

At this point, prior to the results presentation, it is worth highlighting the difference between two loss values that will be presented: real technical loss, that is, the calculated loss from load flow execution in OpenDSS when adding randomly irregular loads to the base system load, according to the location shown in Figure 6; EOI technical loss, that is, the calculated loss using the EOI values shown in Figure 9 obtained for the loading condition considering only the billed demands, after the adjustment of load's power factor, and using the total injected current measurement representing the loading condition with non-technical losses included. Therefore, Figure 10 shows the comparison between the real technical loss and the EOI technical loss curves. It is possible to observe a strong adherence between the two curves at each instant of time, which denotes the high precision of the proposed loss calculation methodology. 


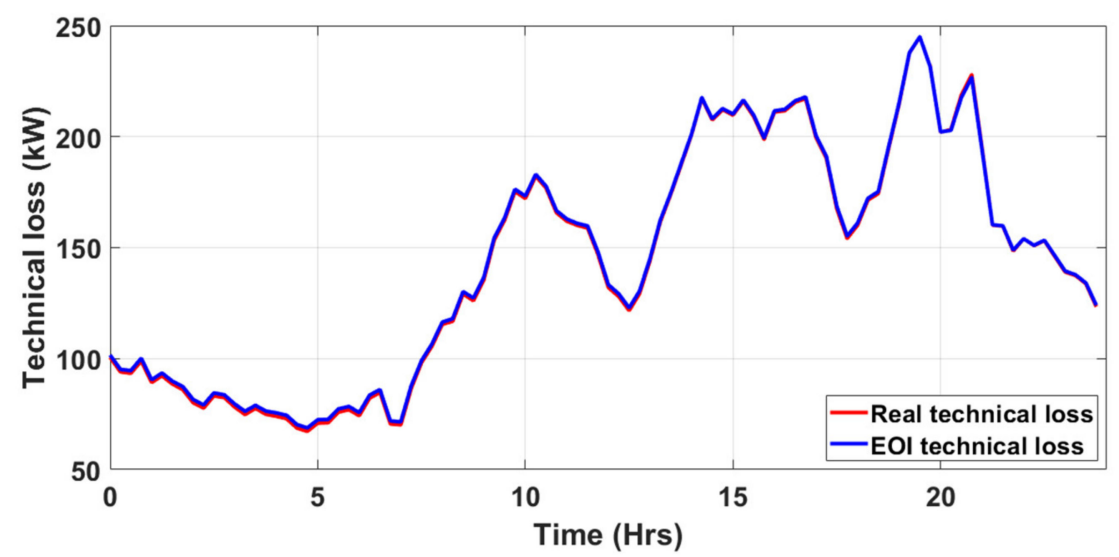

Figure 10. Real technical loss (red) and technical loss calculated using EOI (blue).

Taking the power loss values presented in Figure 10, daily energy losses can be obtained using Equation (17):

$$
e_{T e c}=\sum_{i=1}^{N_{i}} P_{T i} \cdot T
$$

where:

$e_{T e c}$-Energy loss calculated for a day interval in [kWh];

$N_{i}$-Number of time intervals in the day;

$P_{T i}-$ Technical loss for the ith load curve point in the daily cycle $[\mathrm{kW}]$

$T$-Duration of each time interval of the daily load curve [h].

The percentual error between the energy values calculated using the EOI procedure and the load flow routine is calculated as in (18). Considering the curves values presented in Figure 10, an error of $0.5827 \%$ was obtained.

$$
\text { error }=\left(\frac{e_{T e c \_E O I}-e_{T e c \_R e a l}}{e_{\text {Tec_Real }}}\right) \cdot 100
$$

where:

error-Error between the energy values obtained by the two methodologies [\%];

$e_{\text {Tec_EOI }}$-Energy calculated using the EOI methodology [ $\mathrm{kWh}$;

$e_{\text {Tec_Real }}$-Energy calculated using the real technical loss curve $[\mathrm{kWh}]$;

To better evaluate the EOI performance in loss calculation, 1000 load flow simulations were performed, considering random generation for the fraud percentages to be included in the IEEE 123-bus test system base load condition.

As shown in Figure 11a, the average value of non-technical loss added to the base system was $19.42 \%$, with a variation between $16.81 \%$ and $22.03 \%$. On the other hand, the average error obtained between the real loss and the loss calculated by the EOI methodology was $0.7437 \%$, with a variation between $-0.4797 \%$ and $1.9671 \%$. Still considering a normal distribution for the calculated errors, $95.41 \%$ of these values are located in the gray region of Figure 11b, which comprises errors with values in the range specified in (19), which denotes an excellent precision of the proposed methodology.

$$
\text { error }=\mu \pm 2 \cdot \sigma
$$

where:

error-Percentual error;

$\mu$-Error average value;

$\sigma$-Standard deviation; 

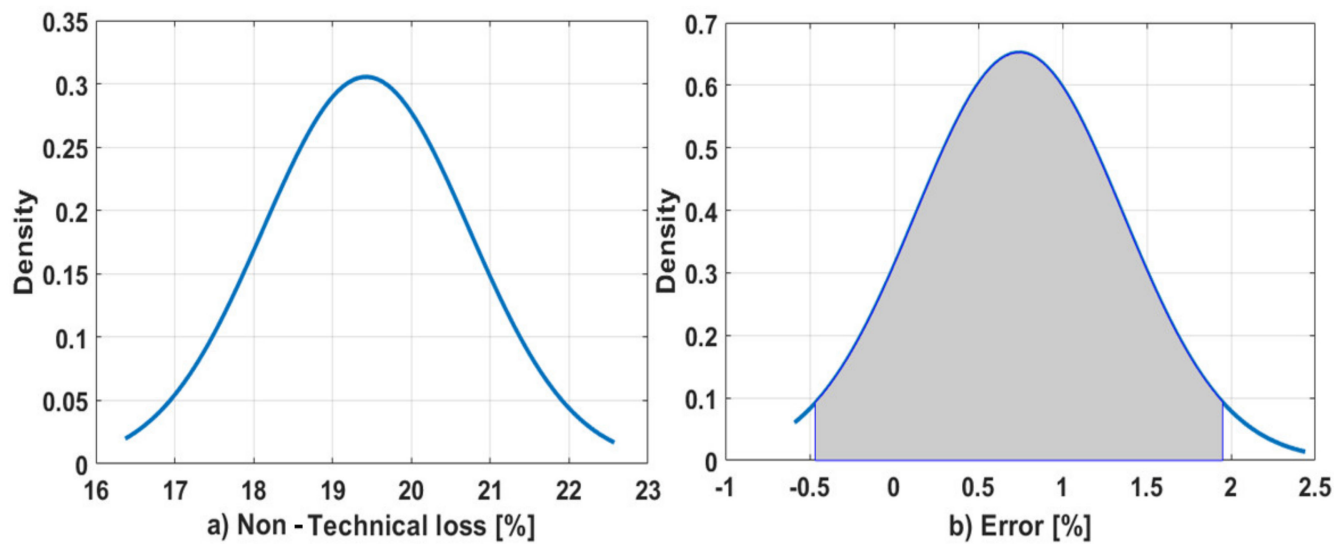

Figure 11. (a) Gaussian distribution of percentages of non-technical loss added to the base system; (b) Gaussian distribution of errors obtained between the real technical loss and the loss calculated by the EOI methodology.

In order to evaluate more scenarios on the percentual values of non-technical loss to be added randomly to the billed load, these percentual values were varied in the ranges of $0-30 \%, 0-20 \%, 0-10 \%$ and $0-5 \%$. Thus, the average total non-technical losses, considering these scenarios and after one hundred simulations were equal to $14.76 \%, 9.76 \%, 4.87 \%$ and $2.44 \%$, respectively. Therefore, for these scenarios, the errors Gaussian curves obtained are shown in Figure 12a-d respectively, for average non-technical losses equal to $14.76 \%$, $9.76 \%, 4.87 \%$ and $2.44 \%$, which resulted in percentual errors equal to $0.6366 \%, 0.5004 \%$, $0.2863 \%$ and $0.1486 \%$ respectively for applying the EOI methodology.
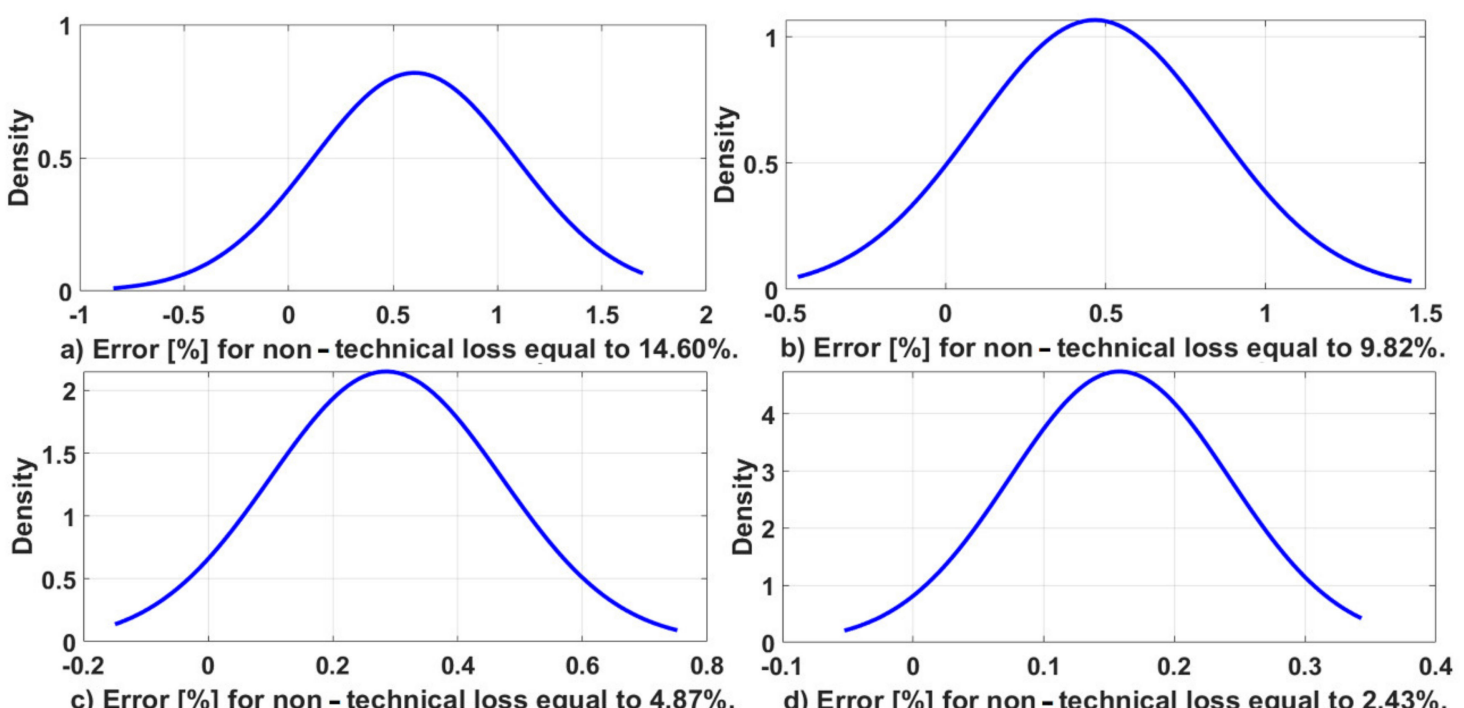

Figure 12. (a) Average percentual error equal to 0.6366\%; (b) Average percentual error equal to 0.5004\%; (c) Average percentual error equal to $0.2863 \%$; (d) Average percentual error equal to $0.1486 \%$.

\subsection{A Real Urban Distribution Substation Case Study}

The real system used in the simulation studies was the Pedreira substation, in the urban area of Belém city in the state of Pará, Brazil, which belongs to the Equatorial Pará, the local distribution utility. This substation has three $20 \mathrm{MVA}, 69 \mathrm{kV} / 13.8 \mathrm{kV}$ transformers supplying 12 distribution feeders, as shown in Figure 13. 


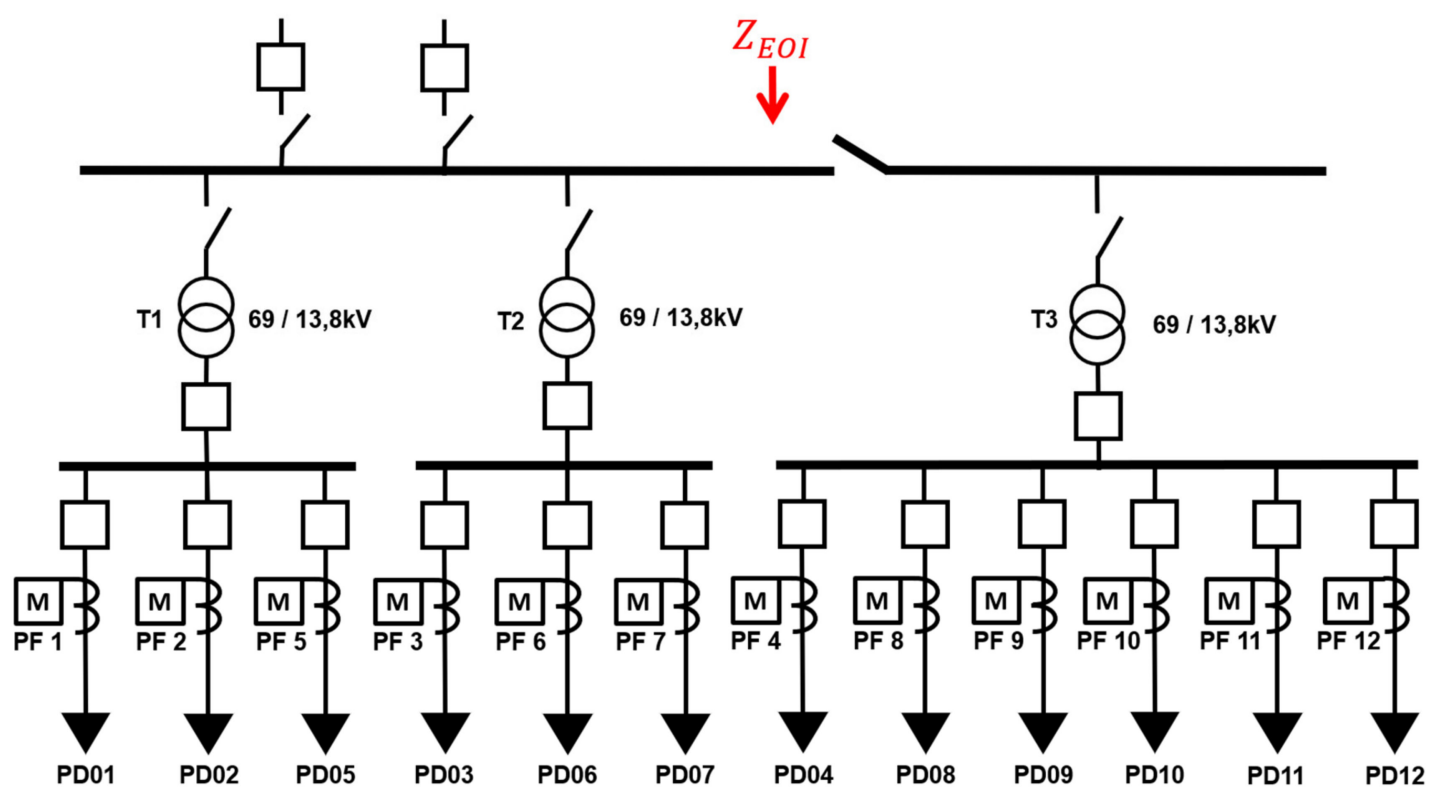

Figure 13. The Pedreira Distribution Substation main configuration.

In Table 3 are presented data to better characterize the substation feeders as: number of low and medium voltage costumers (loads), number of capacitor banks, and lengths of low and medium voltage grids. In addition, at the substation-feeder coupling points, there is measuring devices to measure voltage, injected current, active power and power factor.

Table 3. Substation Pedreira's feeders typical data.

\begin{tabular}{cccccc}
\hline Feeder & $\begin{array}{c}\text { Number of Low } \\
\text { Voltage Loads }\end{array}$ & $\begin{array}{c}\text { Number of } \\
\text { Medium } \\
\text { Voltage Loads }\end{array}$ & $\begin{array}{c}\text { Number of } \\
\text { Capacitor Banks }\end{array}$ & $\begin{array}{c}\text { Length of Medium } \\
\text { Voltage Network } \\
{[\mathbf{k m}]}\end{array}$ & $\begin{array}{c}\text { Length of Low } \\
\text { Voltage } \\
\text { Network [km] }\end{array}$ \\
\hline PD01 & 2368 & 7 & 2 & 7.22 & 70.31 \\
PD02 & 4297 & 22 & 1 & 6.14 & 116.64 \\
PD03 & 7392 & 28 & 2 & 9.42 & 196.61 \\
PD04 & 8721 & 4 & 2 & 9.45 & 226.58 \\
PD05 & 6335 & 16 & 2 & 9.32 & 177.77 \\
PD06 & 5005 & 16 & 2 & 7.25 & 135.38 \\
PD07 & 5833 & 12 & 1 & 6.92 & 157.71 \\
PD08 & 4036 & 1 & 0 & 8.02 & 105.22 \\
PD09 & 3541 & 27 & 2 & 10.13 & 110.75 \\
PD10 & 4517 & 9 & 1 & 6.93 & 120.85 \\
PD11 & 6856 & 14 & 4 & 11.87 & 185.43 \\
PD12 & 7181 & 7 & 1 & 12.24 & 195.26 \\
\hline
\end{tabular}

Using the substation modeled in OpenDSS and measured electrical data the substation coupling point, technical and non-technical loss calculation will be performed using the integrated loss calculation methodology, shown in Figure 5.

Following flowchart in Figure 6, in step 3 of the loss calculation methodology, the load power factor is adjusted, according to the algorithm presented in Figure 3. For this purpose, power factor measurements at the beginning of feeders, were used and then the algorithm was executed for each feeder individually. As an example of application, Table 4 presents the monthly average power factor obtained from measurements at the beginning of feeders and the power factor calculated for the corresponding monthly billed demand by load flow simulation, before applying the power factor correction algorithm. The objective of the power factor correction algorithm is to make these two values equal, by adjusting the low voltage loads' power factors. 
Table 4. Power factors measured in the beginning of feeders and calculated by load flow algorithm.

\begin{tabular}{ccc}
\hline Feeder & $\begin{array}{c}\text { Power Factor Measured at } \\
\text { the Substation-Feeder } \\
\text { Coupling Point }\end{array}$ & $\begin{array}{c}\text { Power Factor Calculated at the } \\
\text { Substation-Feeder Coupling Point before } \\
\text { Running the Correction Algorithm }\end{array}$ \\
\hline PD01 & 0.87 & 0.9999 \\
PD02 & 0.97 & 0.9824 \\
PD03 & 0.93 & 0.9843 \\
PD04 & 0.96 & 0.9982 \\
PD05 & 0.97 & 0.9972 \\
PD06 & 0.92 & 0.9971 \\
PD07 & 0.93 & 0.9622 \\
PD08 & 0.87 & 0.9191 \\
PD09 & 0.96 & 0.9954 \\
PD10 & 0.90 & 0.9602 \\
PD11 & 1.00 & 0.9011 \\
PD12 & 0.95 & 0.9878 \\
\hline
\end{tabular}

As shown in Table 4, a usual practice in most distribution utilities in Brazil is to assume an initial power factor value equal to 0.92 to all low voltage loads. The algorithm of Figure 3 was then executed individually for each feeder, for the average loading condition for one simulation point. Then, after the algorithm execution, different power factor values were obtained for the low voltage loads for each feeder, to be used in step 4 of Figure 5, as presented in Table 5 .

Table 5. Power factor correction algorithm applied at low voltage loads of the feeders.

\begin{tabular}{ccc}
\hline Feeder & $\begin{array}{c}\text { Adopted Power Factor at } \\
\text { Low Voltage Loads }\end{array}$ & $\begin{array}{c}\text { Calculated Power Factor at Low Voltage Loads } \\
\text { after Running }\end{array}$ \\
\hline PD01 Correction Algorithm
\end{tabular}

Therefore, to perform the loss calculation according to the proposed methodology in Figure 5, the adjusted power factor values as presented in Table 5 are used. To illustrate the power factor correction algorithm applied to a daily load cycle, it is presented in Figure 14 the results obtained for PD01 Feeder's loads for weekday, Saturday and Sunday load curve profiles considering only billed demands.

It is evident from observing Figure 14 that adopting a single power factor value to all low voltage loads, as for example 0.92 , may lead to imprecisions when calculating technical and non-technical losses in low voltage distribution grids.

After adjusting the low voltage loads' power factors in step 3, the loss calculation methodology is continued in step 4, by executing load flow cases in OpenDSS, for 96 simulation points for weekday, Saturday, and Sunday. In this step, technical losses due to supplying only billed demands (regular loads) are obtained, as observed in Figure 15a. Then, using this calculated technical loss and the total injected current in the substationfeeder coupling bus, both obtained by the executed load flow cases in OpenDSS, the EOI is obtained in step 5, shown in Figure 15b. Still using the load flow results, the distribution 
grid load curve is obtained in step 6, shown in Figure 15c. Then, using the feeder's monthly injected energy data, obtained from the commercial database, and having the load curve obtained in step 6, the feeder's total injected power curve is obtained in step 9, shown in Figure $15 \mathrm{~d}$, which include regular and irregular loads, that is, billed and non-billed power.

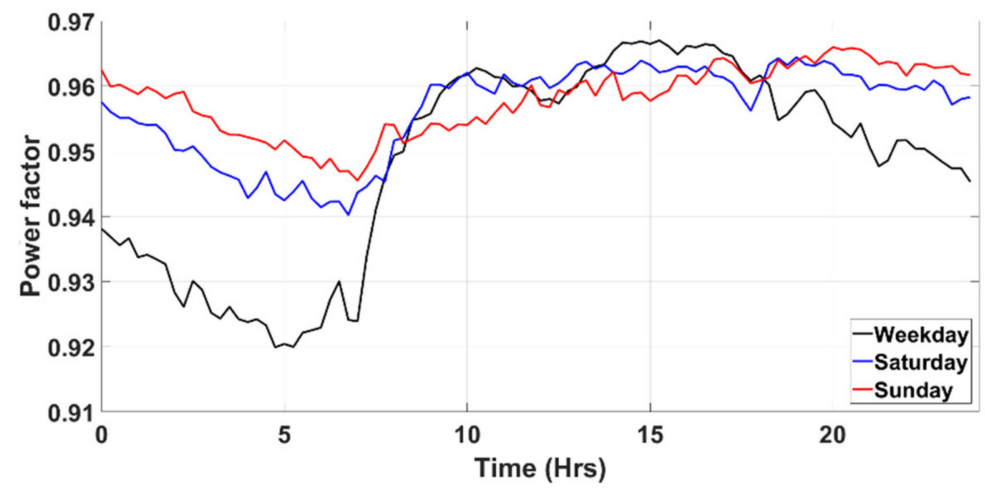

Figure 14. Power factor daily variation calculated at the PD01 feeder-substation coupling point for a weekday (black), Saturday (blue) and Sunday (red), load curve profiles for billed demands only.
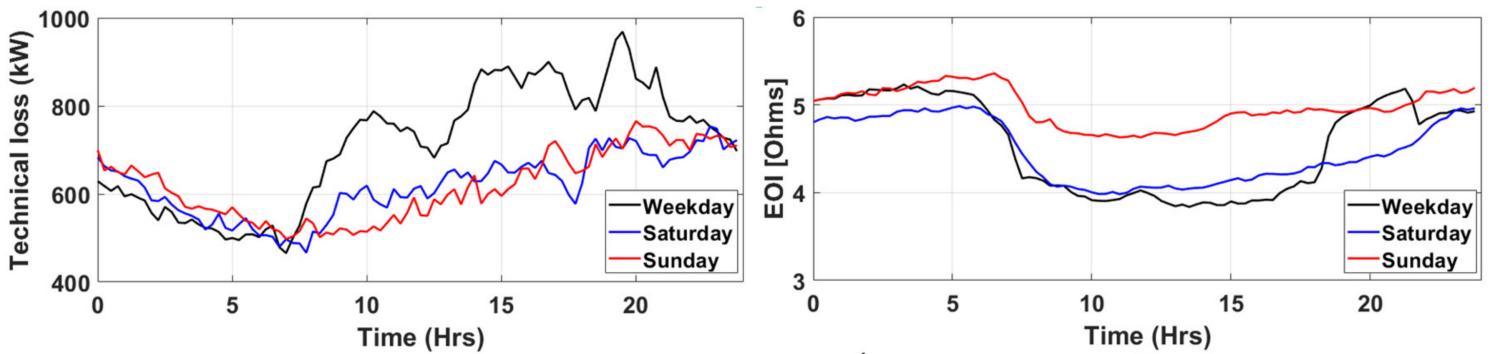

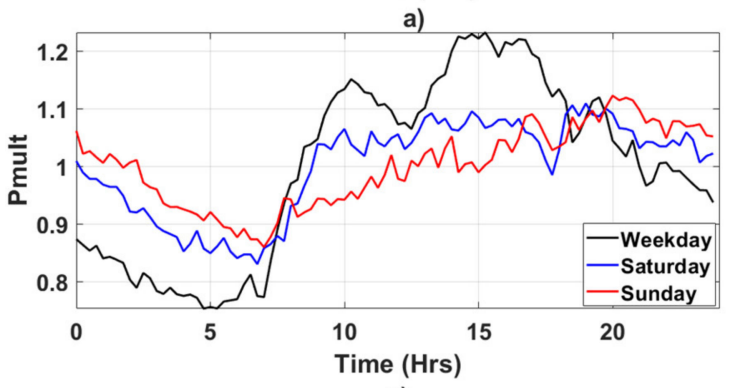

c)

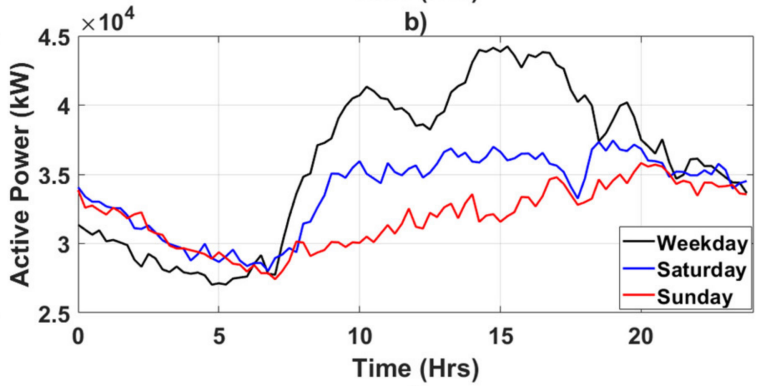

d)

Figure 15. (a) Technical loss curve due to the regular loads supply; (b) Resistive part of EOI; (c) Distribution feeder's loading curve due to billed power only; (d) Total feeder's injected power curve due to billed and non-billed power.

Continuing the methodology, step 11 accomplishes the calculation of total injected current into the feeder's electric network, using the measured injected power curve, given in Figure 15d, measured voltage and power factor curves as shown in Figure 16a,b.

Thus, using the total measured injected current and the EOI calculated in Figure 15b, the total technical loss curve can be obtained, as shown in Figure 17. This calculated technical loss comprises losses resulting from supplying regular and irregular loads as well as other sources of non-technical losses that may have occurred in the electrical network under analysis.

After calculating total technical losses, the methodology is continued to calculate the non-technical losses. For this, the data of monthly energy consumed by each consumer unit are extracted and arranged as demand curves for a weekday, a Saturday and a Sunday, in step 13, as shown in Figure 18a. Then, in step 14, the total loss is calculated by subtracting total injected power curves and power demand curves, shown in Figure 18b. 


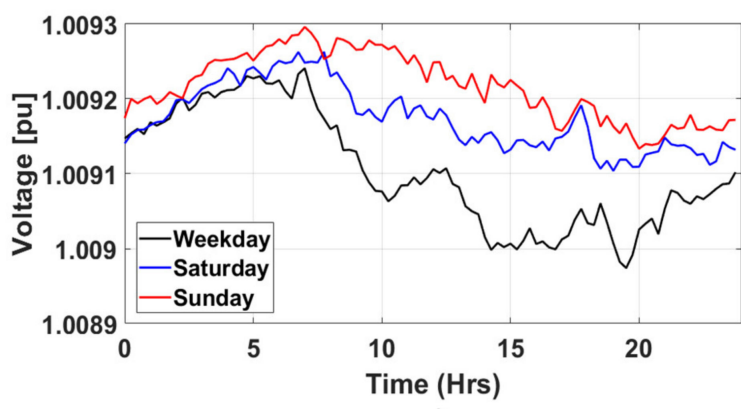

a)

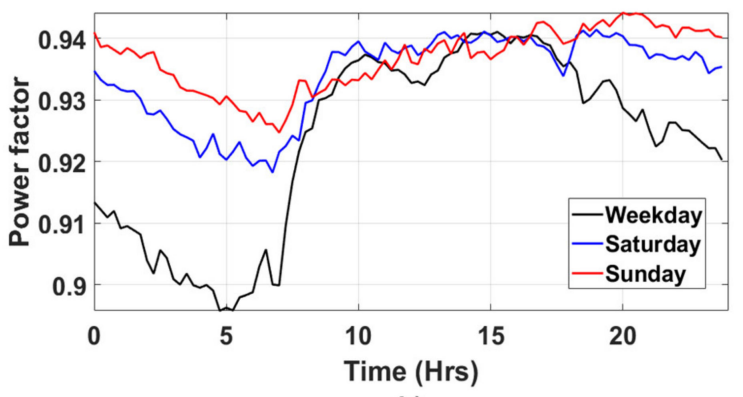

b)

Figure 16. (a) Measured voltage curve at the substation-feeder coupling bus; (b) Measured power factor curve at the substation-feeder coupling bus.

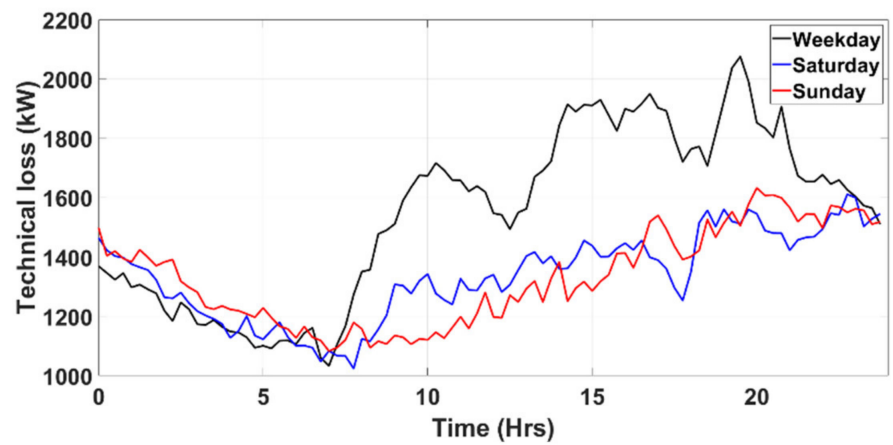

Figure 17. Feeder's total active technical loss curves, which includes technical losses due to supplying regular and irregular consumers and other sources of non-technical losses.

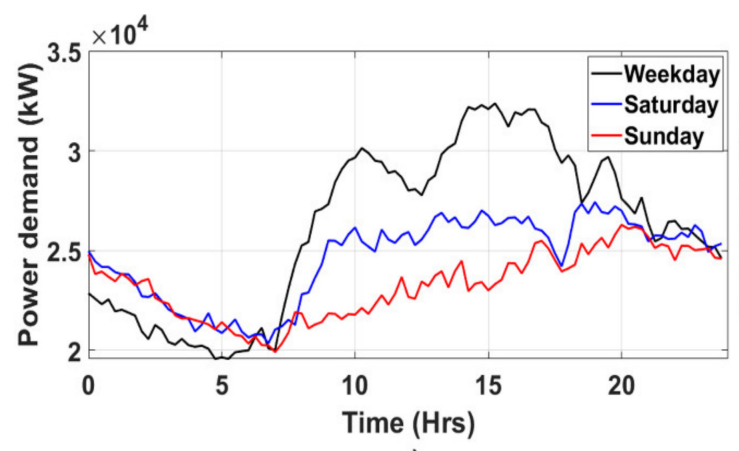

a)

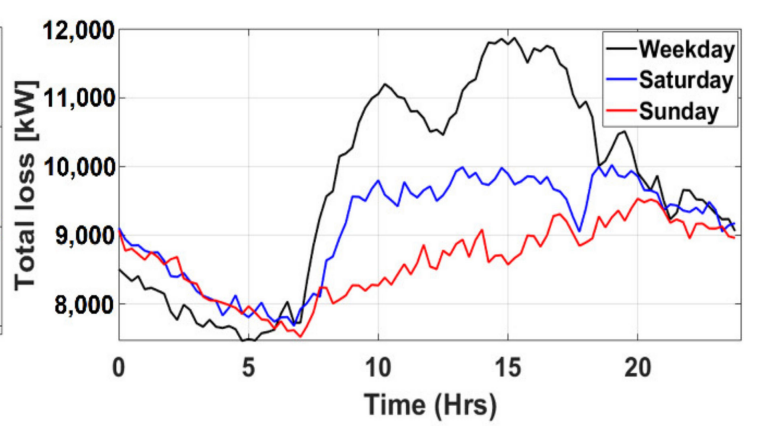

b)

Figure 18. (a) Power demand curve; (b) Total loss curve.

Finally, in step 15, the electric network non-technical losses are calculated, by subtracting the total losses from the total technical losses, as shown in Figure 19.

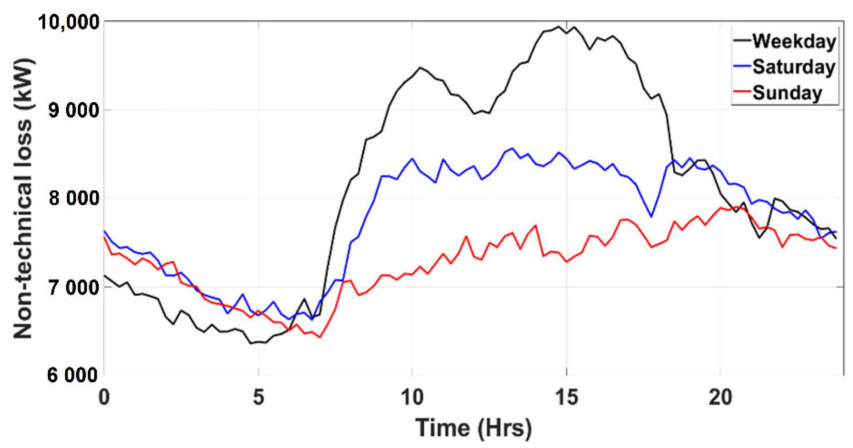

Figure 19. Non-technical losses for weekday (black), Saturday (red) and Sunday (blue). 
Aiming to obtain the loss data calculated using the methodology in terms of energy, the daily curves can be integrated to obtain daily, weekly, monthly, and annual energy losses data.

\section{Conclusions}

In this article, two innovative procedures were highlighted in relation to the systematic calculation of technical and non-technical losses in electrical distribution networks. The first refers to using an Equivalent Operational Impedance (EOI) for representing the electrical network in calculating technical losses. It was demonstrated by the results presented that the use of EOI allows the total losses, which include technical and non-technical losses, to be obtained for each operating condition of the distribution network under analysis, simply by multiplying the squared rms value of the current injected in the feeder, by the operational resistances and reactances, resulting in the respective network active and reactive losses, with minimal computational effort. This feature undoubtedly highlights the use of EOI for real-time operation of electrical distribution networks, which will be approached in future works.

For other applications in technical and non-technical losses management, the joint use of EOI with the proposed procedure of adjusting the power factor of customers' loads, so that the power factor calculated in the substation bus meets the measured value at this point, makes the loss calculation procedure more accurate. Also, the proposed loss calculation procedure allows the breakdown of technical and non-technical losses for each operating condition of the electrical grid, which will certainly contribute to a better management of these losses.

The results obtained in calculating technical losses in the IEEE 123-bus system showed the accuracy of the proposed methodology considering different levels of non-technical losses included randomly in network load buses.

The methodology was also applied in a real distribution system, using real operational data obtained from field measurements. The results presented in terms of technical and non-technical losses, voltages, power factors, and demands are adequately detailed to characterize the electrical distribution grid operational conditions, for a daily cycle of operation, involving typical load curves for weekdays, Saturdays, and Sundays. The results were presented by substation, discretized at 15 min intervals, which constitutes relevant information for the distribution grid short term operation planning.

Considering the implementation of the proposed methodology in a real distribution system, some important data must be collected and maintained continuously by utilities, such as: a georeferenced technical database containing updated information on electrical cables, transformers, capacitor banks, loads and other network elements that are part of the electric network topology, and data informing the electrical connections among them for composing mathematical models in OpenDSS; typical load demands curves describing the loads' consumption cycles for weekdays, Saturdays, and Sunday; a commercial database to inform the monthly customers' energy bills; power factor measurement data at feeders coupling points at the substation. If the electric utility does not have available these data or part of it, this may be a limitation to implement the proposed methodology.

As a continuation of this work, there is a proposal to implement a real time application of the proposed methodology to calculate technical losses for every measured real time injected electric current at the distribution feeders. For such implementation, the EOI calculation will be coupled to the output of a real time network configurator program, so that for each topology modification a new EOI is obtained. This proposal is suited to be implemented in a distribution network management system, obtaining technical losses for each feeder at the coupling point with the substation.

In addition, other operating scenarios will be evaluated in test systems, in order to evaluate the loss calculation accuracy in such cases as: system operating with under and over voltage; system operating with and without voltage regulators; insertion of small 
and medium-sized distributed generation; and uneven distribution of non-technical losses between different feeders or parts of the system, which often occurs in real systems.

Author Contributions: Conceptualization, C.E.M.R., M.E.d.L.T., and U.H.B.; methodology, C.E.M.R., M.E.d.L.T. and U.H.B.; data acquisition, L.S.S.F., E.C.d.S.S., M.F.R. and C.J.d.S.M.; network modeling, T.M.S. and C.E.M.R.; software implementation, T.M.S. and C.E.M.R.; results analysis, C.E.M.R., U.H.B., E.O.d.M., L.S.S.F., E.C.d.S.S., M.F.R. and C.J.d.S.M.; writing-review and editing, C.E.M.R., M.E.d.L.T., U.H.B., T.M.S. and E.O.d.M.; supervision, M.E.d.L.T., U.H.B., L.S.S.F., E.O.d.M., E.C.d.S.S., M.F.R. and C.J.d.S.M. All authors have read and agreed to the published version of the manuscript.

Funding: This research received no external funding.

Institutional Review Board Statement: Not applicable.

Informed Consent Statement: Not applicable.

Data Availability Statement: The data presented in this study are available on request from the corresponding author. The data are not publicly available due to the existence of data from the energy company.

Conflicts of Interest: No conflict of interest.

\section{References}

1. Sallam, A.A.; Malik, O.P. Electric Distribution Systems, 1st ed.; John Wiley \& Sons: Hoboken, NJ, USA, $2011 ;$ pp. 17-18.

2. ANEEL, Energy Losses in Electric Distribution Networks. Available online: https://www.aneel.gov.br/documents/654800/18766 993/Relat\%C3\%B3rio+Perdas+de+Energia_+Edi\%C3\%A7\%C3\%A3o+1-2019-02-07.pdf/d7cc619e-0f85-2556-17ff-f84ad74f1c8d (accessed on 15 December 2019).

3. Bezerra, U.H.; Soares, T.M.; Nunes, M.V.A.; Tostes, M.E.L.; Vieira, J.P.A.; de Oliveira, R.C. Estimating Technical and NonTechnical Losses in Electrical Distribution Networks Using Power Measurement at the Substation and Customers' Energy Billing Information. In Proceedings of the 6th Brazilian Symposium on Electrical Systems, Natal, Rio Grande do Norte, Brazil, 23 May 2016.

4. $\quad$ Ramesh, L.; Chowdhury, S.P.; Chowdhury, S.; Natarajan, A.A.; Gaunt, C.T. Minimization of Power Loss in Distribution Networks by Different Techniques. Int. J. Electr. Comput. Eng. 2009, 3, 4.

5. Figueiredo, G.A.D. Characterization of Losses in the Medium Voltage Distribution Network. Master's Thesis, Faculty of Engineering, University of Porto, Porto, Portugal, 2012.

6. Henriques, H.O.; Corrêa, R.L.S. Use of Smart Grids to Monitor Technical Losses to Improve Non-Technical Losses Estimation. In Proceedings of the 7th Brazilian Symposium on Electrical Systems Rio de Janeiro, Rio de Janeiro, Brazil, 12-16 May 2018.

7. Agüero, J.R. Improving the Efficiency of Power Distribution Systems through Technical and Non-Technical Losses Reduction. In Proceedings of the IEEE PES Transmission \& Distribution (T\&D), Orlando, FL, USA, 7 May 2012.

8. Queiroz, L.M.O.; Roselli, M.A.; Cavellucci, C.; Lyra, C. Energy Losses Estimation in Power Distribution Systems. IEEE Trans. Syst. 2012, 27, 4. [CrossRef]

9. Oliveira, C.C.B.; Kagan, N.; Meffe, A.; Caparroz, S.L.; Cavaretti, J.L. A New Method for the Computation of Technical Losses in Electrical Power Distribution Systems. In Proceedings of the CIRED, Amsterdam, The Netherlands, 18-21 June 2001.

10. Meffe, A. Methodology for Calculating Technical Losses by Segment in Electrical Distribution Systems. Master's Thesis, Politecnica School, University of Sao Paulo, Sao Paulo, Brazil, 2001.

11. Oliveira, C.C.B.; Kagan, N.; Méffe, A.; Jonathan, S.; Caparroz, S.L.; Cavaretti, J.L. Calculation of Energy Technical Losses and Demand by Segment in Electrical Distribution Systems. In Proceedings of the XIV SENDI, Foz do Iguaçu, Brazil, 19-23 November 2000.

12. Schmidt, H.P.; de Oliveira, C.C.B.; Méffe, A.; da Rosa, M.A. Loss Estimation in LV Circuits Using Intelligent Techniques-The RGE Experience. In Proceedings of the CIRED 2003, Barcelona, Spain, 12-15 May 2003.

13. Liu, H.; Bai, Y.; Liang, M.; Chen, H.; Ren, X. Research of State Monitoring and Pre-warning System of Cable Joint in Distribution power grids. In Proceedings of the International Conference on Power System Technology, Chongqing, China, 22-26 October 2006.

14. Nikovski, D.; Wang, Z.; Esenther, A.; Sun, H.; Sugiura, K.; Muso, T.; Tsuru, K. Smart Meter Data Analysis for Power Theft Detection. Mach. Learn. Data Min. Pattern Recognit. Lect. Notes Comput. Sci. 2013, 7988, 379-389.

15. Zhang, Q.; Shi, K.; Zhang, J. New features for detection of nontechnical losses considering PV installed at customer side. In Proceedings of the China International Conference on Electricity Distribution (CICED), Shanghai, China, 10-14 September 2012.

16. Huang, S.; Lo, Y.; Lu, C. Non-technical loss detection using state estimation and analysis of variance. IEEE Trans. Power Syst. 2013, 28, 2959-2966. [CrossRef]

17. Kaykahie, S.; Kowsari, S. A new approach for calculating load and loss factor based on consumer data with fuzzy modelling. In Proceedings of the 22nd International Conference on Electricity Distribution, Stockholm, Sweden, 10-13 June 2013. 
18. Bezerra, U.H.; Soares, T.M.; Vieira, J.P.A.; Tostes, M.E.L.; Manito, A.R.R.; Paye, J.C.H. Equivalent operational impedance: A new approach to calculate technical and non-technical losses in electric distribution systems. In Proceedings of the Brazilian Symposium on Electrical Systems (SBSE), Niteroi, Brazil, 12-16 May 2018.

19. Paye, J.C.H. Technical and Non-Technical Losses Calculation in Electrical Distribution Networks Using the Definition of Equivalent Operational Impedance. Master's Thesis, Federal University of Pará, (UFPA), Belém, Brazil, 2018.

20. Bezerra, U.H.; Soares, T.M.; Vieira, J.P.A.; Nunes, M.V.; Tostes, M.E.; de Oliveira, R.C. Technical and non-technical losses calculation in distribution grids using a defined equivalent operational impedance. IET Gener. Transm. Distrib. 2019, 13, $1315-1323$.

21. Costa, S.C.P.; Rodrigues, C.E.M.; Tostes, M.E.L.; Bezerra, U.H.; Filho, L.S.S.; Medeiros, V.G. Technical and non-technical losses calculation in distribution grids: A comparative analysis between the ANEEL recommended methodology and using the equivalent operational impedance. In Proceedings of the XIII Brazilian Conference on Electric Power Quality, São Paulo, Brazil, 1-4 September 2019.

22. Cruz, E.C. Analysis of Methodological Procedures for Georeferencing and Registration of Attributes of Urban Electrical Networks in Compliance with ANEEL's Standards. Master's Thesis, São Paulo State University in President Prudente City, São Paulo, Brazil, 2013.

23. Au, M.T.; Anthony, T.M.; Kamaruddin, N.; Verayiah, R.; Mustaffa, S.A.S.; Yusoff, M. A Simplified Approach in Estimating Technical Losses in Distribution Network Based on Load Profile and Feeder Characteristics. In Proceedings of the 2th IEEE International Conference on Power and Energy, Johor Baharu, Malaysia, 1-3 December 2008.

24. Wu, A.; Ni, B. Line Loss Analysis and Calculation of Electric Power Systems; Wiley \& Sons: Beijing, China, 2016.

25. Moura, P.R.F.; Souza, B.A. Proposal of a Simplified Methodology for Calculating Technical Losses. In Proceedings of the XVIII National Seminar on Electric Power Distribution, Olinda, Brazil, 6-10 October 2008.

26. Almeida, M.A.D.; Silveira, D.B.F.; Júnior, M.F.M. Estimating Loads in distribution Feeders Using a State Estimation Algorithm with Additional Adjustment of Transformers Loading Factors. In Proceedings of the International Symposium on Circuits and Systems, Bangkok, Thailand, 25-28 May 2003.

27. Grainger, J.J.; Kendrew, T.J. Evaluation of Technical Losses on Electric Distribution Systems. In Proceedings of the 10th International Conference on Electricity Distribution (CIRED), Brighton, UK, 8-12 May 1989.

28. Fan, Y.; Jun, L.; Wenrong, S.; Li, X. Research on Theoretical Line Loss Calculation of Low-Voltage Power Distribution Area in Shanhai. In Proceedings of the China International Conference on Electricity Distribution (CIDED), Shenzhen, China, 23-26 September 2014.

29. Dashtaki, A.K.; Haghifam, M.R. A New Loss Estimation Method in Limited Data Electric Distribution Networks. IEEE Trans. Power Deliv. 2013, 28, 4. [CrossRef]

30. Kersting, W.H. Radial distribution test feeders. In Proceedings of the IEEE Power Engineering Society Winter Meeting, Columbus, OH, USA, 28 January-1 February 2001. 